\title{
Síndrome de Burn-out: um olhar para o esgotamento profissional do docente universitário
}

\author{
Burn-out Syndrome: A look into the \\ professor's professional exhaustion
}

\author{
Elizabete Cazzolato Ferreira ${ }^{1}$ \\ Julia Alejandra Pezuk ${ }^{2}$
}

Resumo: As dimensões do estresse no trabalho repercutem sobre a saúde dos indivíduos podendo causar distúrbios que impactam na qualidade de vida e/ou produtividade. As disfunções emocionais possuem traços comuns que permitem estudar as variáveis que compõem a Síndrome de Burn-out. Esse estudo procurou compreender esse tema no universo da docência de ensino superior a partir da produção científica dos últimos cinco anos. Os resultados evidenciaram a preocupação com o adoecimento silencioso do docente, buscando o entendimento clínico das repercussões do estresse a partir da aplicação de testes validados e da identificação dos potenciais causadores do desequilíbrio emocional que acomete a qualidade de vida a partir das atividades laborais. Na tentativa de compreender a vulnerabilidade, houve destaque para recomendações preventivas em grupos com maior propensão ao Burn-out, citando, dentre esses, as docentes com idades extremas ou do sexo feminino. Os influenciadores externos (relacionados ao ambiente acadêmico, cultura organizacional e alta demanda de trabalho com tarefas diversificadas), bem como as propensões pessoais (como enfermidades psicológicas prévias, resiliência e espiritualidade) são abordados como aspectos importantes para o enfrentamento, e como propostas de intervenção. A contextualização da Síndrome de Burn-out em docentes universitários abarca campos de ordem pessoal, filosófica, psicológica, cultural e organizacional. Assim, é possível concluir que as propostas de intervenção e controle ainda são subjetivas, o que é influenciado pelo fato de que a Síndrome ainda não é reconhecida como uma doença, e de que a maioria das pesquisas busca entender as proporções e características dessa condição.

Palavras-chave: Estresse ocupacional. Esgotamento psicológico. Professor. Saúde mental. Ensino. 
Abstract: The emotional aspects related to work stress have consequences on individual's health and may impact on the quality of life and/or productivity. Emotional dysfunctions have common features that allow the study of variables associates to Burn-out Syndrome. This study sought to understand this theme on higher education teaching using the scientific production of the last five years. The results evidenced the concern with the professor's silent sickness, seeking the clinical understanding of stress's repercussions from the application of validated tests and the identification of the potential causes of the emotional imbalance that affects the quality of life from the work activities. To understand the vulnerability, there was a highlight for preventive recommendations in groups with a greater propensity for Burn-out, mentioning, among those, professors with extreme ages or females. External influencers (related to the academic environment, organizational culture, and high demand of work with diverse tasks), as well as personal propensities (such as previous psychological illnesses, resilience, and spirituality), were addressed as important aspects for coping and as intervention proposals. It is showed that Burn-out Syndrome in university professors encompasses fields of a personal, philosophical, psychological, cultural, and organizational order. Thus, it is possible to conclude that the proposals for intervention and control are still subjective, which is influenced by the fact that the Syndrome is not yet recognized as a disease, and that most research seeks to understand the proportions and characteristics of this condition.

Keywords: Occupational Stress. Psychological burnout. Teacher. Mental health. Teaching.

\footnotetext{
${ }^{1}$ Universidade Anhanguera de São Paulo | Programa de Pós-graduação em Ciências e Saúde | São Paulo | SP | Brasil. Contato: elizabete.cazzolato@gmail.com. ORCID: https://orcid.org/0000-0003-2045-6489

${ }^{2}$ Universidade Anhanguera de São Paulo | Programa de Pós-graduação em Ciências e Saúde | São Paulo | SP | Brasil. Contato: julia.pezuk@hotmail.com. ORCID: https://orcid.org/0000-0001-5412-6619
}

- Recebido em: 9 de julho de 2020

- Aprovado em: 9 de abril de 2021

DOI: http://dx.doi.org/10.1590/S1414-40772021000200008

Este é um artigo publicado em acesso aberto sob uma licença Creative Commons https://creativecommons.org/licenses/by-nc/4.0/ 


\section{Introdução}

Na dialética do exercício docente, entre a consciência do executar e o limite do realizar, observamos uma variedade e quantidade infinita de estímulos, informações, decisões, excessivas tarefas e obrigações que, sem que se perceba, vão se acumulando numa rotina de trabalho exaustiva em termos de produtividade e esforço mental para superação de demandas. A tentativa de atender todas as exigências profissionais, associada à traços pessoais, pode levar os indivíduos ao desenvolvimento de um quadro sintomático de esgotamento e exaustão emocional com repercussões físicas. Bernardes (2018) destaca que o aumento da intensidade e pressão num mundo cada vez mais competitivo, rápido e produtivo, adicionado aos conflitos interpessoais frequentemente encontrados nos locais de trabalho, ao desequilíbrio entre expectativa e realidade, às frustrações profissionais e ao mal uso do tempo, têm favorecido o aparecimento de algumas condições clínicas prejudiciais para a saúde do ser humano.

Dados divulgados no início de 2020 pela Isma-BR (International Stress Management Association no Brasil) demonstram o panorama do esgotamento profissional no país, descrevendo que grande parte dos trabalhadores brasileiros se sente incapacitado para o trabalho. De fato, tem sido observado que cerca de $72 \%$ da população economicamente ativa apresenta alto índice de estresse, e pelo menos 30\% desses podem ser enquadrados com a Síndrome de Burn-out (FILIPPI; BOMFIM, 2020). Trabalhadores de distintas áreas sofrem diretamente os efeitos das circunstâncias laborais no que tange a crescentes desafios e novas perspectivas, não apenas no campo profissional, mas também no âmbito social, econômico e tecnológico. Existem alguns tipos de atividades, principalmente aquelas cujos trabalhadores prestam assistência ou são responsáveis pelo desenvolvimento ou cuidado de outros, que expõem os indivíduos a um desgaste emocional maior (PRADO, 2016). E, muito embora as organizações sejam as protagonistas da geração e permissão de um cenário que adoece seus funcionários, esses mesmos empregadores sofrem com o alto absenteísmo, baixo desempenho e produtividade do grupo afetado. Numa referência inversamente proporcional, além de diminuir o rendimento profissional, os problemas de saúde ocasionados por estresse no trabalho aumentam a rotatividade voluntária, gerando mais custos, além de um ambiente tóxico e desmotivador para a empresa.

A atividade docente é considerada pela Organização Internacional do Trabalho como uma das profissões cuja incidência de elementos associados ao estresse é cada vez mais evidente (BATISTA et al., 2010; PRADO, 2016; PFEFFER, 2019). Segundo Massa et al. (2016), os docentes aparecem como profissionais muito vulneráveis à síndrome de Burn-out, sendo que a 
maioria das pesquisas enfocam nos professores do ensino infantil e médio, havendo menos estudos direcionados aos professores de ensino superior. Num panorama sobre contextos da educação superior nacional, Diniz e Goergen (2019), sintetizam uma investigação que destaca a percepção dessa modalidade de ensino como uma das alavancas de desenvolvimento social, econômico, cultural e científico. Esses múltiplos papéis geram um acréscimo de responsabilidades, tarefas e prioridades, que levou a universidade a direcionar os docentes à priorização de atividades em decorrência de metas, indicadores e critérios de avaliação da qualidade do ensino para possibilitar a manutenção, a ascensão e a estabilidade na carreira acadêmica.

O termo Burn-out surgiu a partir das observações do médico alemão Freudenberger em 1974, que acompanhou o processo de desgaste de trabalhadores de uma clínica de reabilitação de dependentes químicos, até a caracterização do quadro de exaustão, definido pela presença de sintomas deletérios tanto de ordem física quanto psíquica (CARLOTTO, 2002). A contextualização de síndrome perpassa pela complexidade que tange a etiologia e a manifestação do estado máximo de esgotamento mental e físico causada por questões relacionadas ao trabalho, de forma que a Síndrome de Burn-out pode ser considerada uma conjunção tridimensional de fatores interligados às atividades laborais e geradores de um colapso de sentimentos (esgotamento emocional), frustração em relação ao senso de produção e desempenho (realização) e perda de identidade profissional (despersonalização) (BAPTISTA et al., 2019; CARLOTTO, 2002; MASSA et al., 2016).

De acordo com a Organização Pan Americana de Saúde (OPAS, 2019), a síndrome não é classificada como doença ou condição de saúde, mas um fenômeno ocupacional que mereceu detalhamento na $11^{\mathrm{a}}$ Revisão da Classificação Internacional de Doenças (CID-11), publicada em abril de 2019. Na vida profissional contemporânea, o estresse, precursor básico da Síndrome de Burn-out, é vivido como uma parte quase inevitável, e, na área docente, isso não parece ser diferente (FERREIRA; PEZUK, 2019). Associada a uma gama de emoções positivas relacionadas aos valores essenciais de quem escolhe a docência de adultos como profissão, a atividade docente mescla uma fonte de realização, satisfação e prazer com elementos de grande impacto emocional como alta pressão, demandas extrassala, sobrecarga de tarefas, estresse em relacionamentos e ritmo acelerado de prazos.

Santos Filho e Dias (2016) referem-se à atividade docente de ensino superior como um processo com sobreposições de atividades, no qual se adiciona a expectativa de que ela seja o palco das inovações em termos de conhecimento, formação e pesquisa. Ao abordar a questão do sofrimento e adoecimento dos docentes de ensino superior, remetemo-nos às suas dimensões 
afetivas, éticas e políticas entrepostas à gama de atividades das universidades contemporâneas, processo esse que se baliza em emoções diversas vezes antagônicas, transitando entre sentimentos positivos e negativos, permeados de realização e frustração (SILVA, 2015). A primeira referência na literatura acerca do impacto do estresse no trabalho docente foi há cerca de 40 anos atrás e descrevia a situação como um fenômeno que gerava emoções negativas resultantes de experiências vivenciadas durante a atividade de trabalho, culminando em estados de raiva, inquietação, tristeza e tensão. Desde 1980, o tema estresse passou a ser considerado mundialmente pela Organização Mundial da Saúde como um ponto de atenção para a redução do bem-estar e qualidade de vida associado ao trabalho, oficializando o impacto emocional e comportamental do desequilíbrio mente e corpo (DALAGASPERINA; MONTEIRO, 2016).

Atualmente, a dinâmica e novos modelos de trabalho do professor universitário, pautados em metodologias ativas, com uso de recursos e controles tecnológicos, têm trazido inovações e demandas que exigem criatividade e atualização constantes. A preocupação com a saúde dos docentes envolve a necessidade de reconhecer os fatores que o levam ao adoecimento, assim como as repercussões de seu estado de saúde na efetividade de suas ações e na relação aluno-professor. Como um mediador do conhecimento, a função de docência superior exige a criação de vínculos de ordem afetiva e emocional, para que a aproximação e a afetividade possam facilitar a construção do aprendizado (BARBOSA, 2016). Impelidas à realização desse estudo, também, por razões científicas e considerando que há pouca literatura sobre o assunto no Brasil, como objetivo dessa pesquisa, buscamos refletir sobre a abordagem da exaustão física e emocional - que caracterizam a Síndrome de Burn-out - no professor universitário divulgada na produção científica dos últimos anos, e analisar os contextos relacionados à mesma.

\section{Metodologia}

Esta pesquisa corresponde a um estudo descritivo, realizado por meio de uma revisão integrativa da literatura científica publicada no período de janeiro de 2015 a maio de 2020 nas bases de dados SCIELO - Scientific Eletronic Library Online (https://scielo.org) e PubMed (https://pubmed.ncbi.nlm.nih.gov) sobre o tema Burn-out em professores de ensino superior. O levantamento foi realizado por meio eletrônico, nos sites das referidas plataformas, utilizando a busca avançada e o operador booleano "and" no título e resumo dos trabalhos, para combinar os descritores em inglês "Burnout" e "Faculty", conforme terminologia citada nos Descritores 
em Ciências da Saúde (DeCS). Dessa forma, procurou-se que cada resultado apresentasse de forma conjunta em seu título ou resumo, as palavras-chave definidoras da pesquisa.

A busca resultou em 218 artigos, sendo 9 na base dados SciELO e 209 na Pubmed. Os títulos e resumos dos trabalhos foram analisados e selecionados à luz dos seguintes critérios de inclusão: publicações nos idiomas inglês, português e espanhol, realizadas nos últimos cinco anos e que apresentassem os descritores inseridos no título ou resumo, contextualizando-os de forma pertinente ao objetivo dessa pesquisa. A figura 1 representa o fluxo de seleção dos artigos, de acordo com os critérios de identificação, seleção, inclusão e exclusão e elegibilidade, preestabelecidos no processo de análise ao qual foram submetidos.

Figura 1 - Fluxo de análise. Seleção dos artigos para realização da revisão de literatura

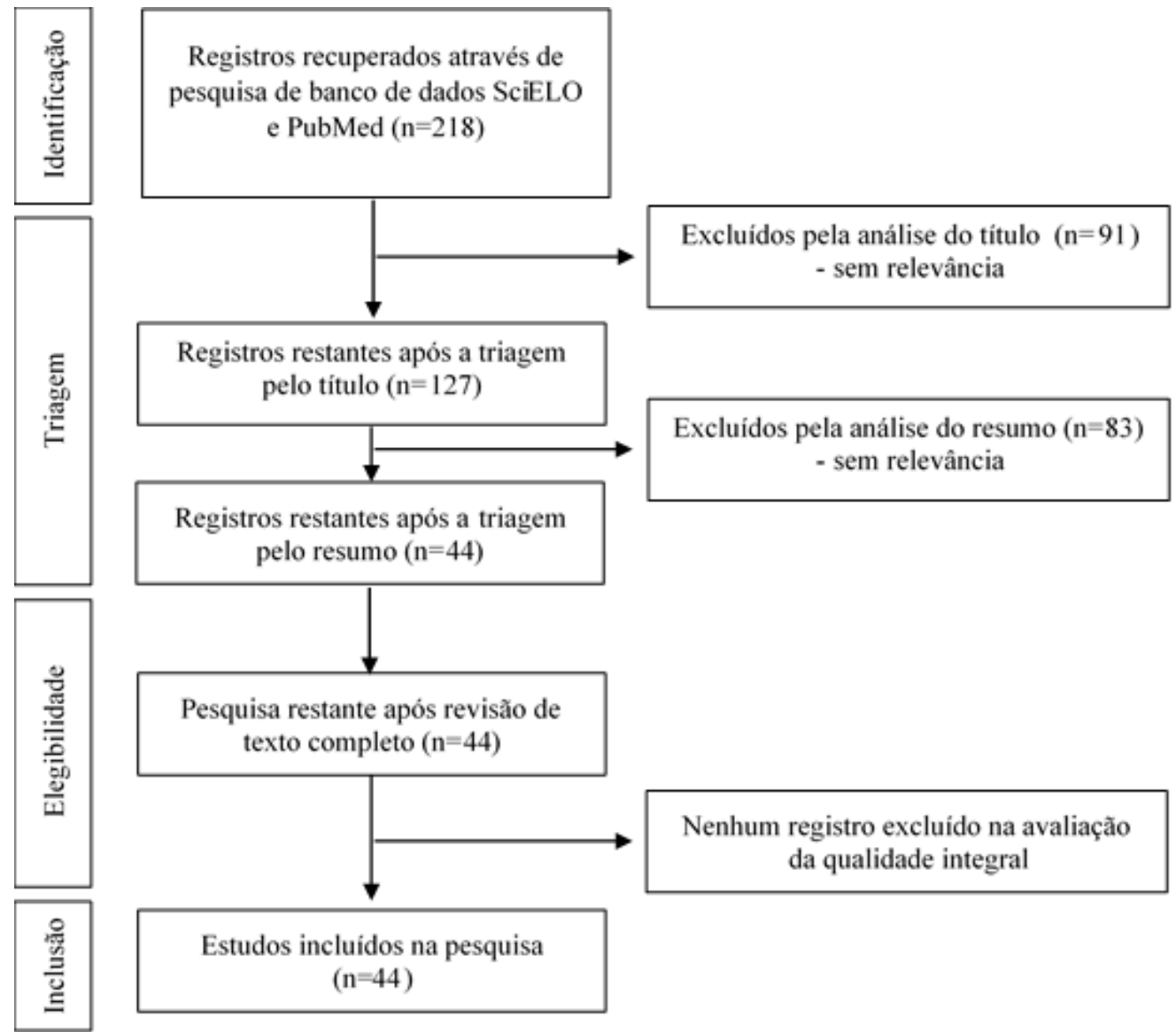

Fonte: Autor

Neste estudo, foram excluídos os artigos publicados em outros idiomas e os que, após leitura e análise do resumo, não apresentavam relevância ao tema abordado (Burn-out em docentes universitários). Posteriormente, as pesquisas selecionadas foram acessadas na íntegra e a análise final foi realizada pela sua relevância, dentro do tema elementar, após a leitura do 
texto integral. Depois da triagem, foram incluídos 44 estudos que atenderam a todos os critérios de inclusão, sendo 4 da base de dados SciELO e 40 da Pubmed. Os 174 artigos excluídos abordavam aspectos da síndrome de Burn-out relacionados a públicos específicos como: estudantes em saúde, residentes, docentes de educação básica, cientistas biomédicos, treinadores físicos, estagiários em saúde, estudantes de engenharia e profissionais da saúde. Após a finalização da triagem, os artigos selecionados foram catalogados e analisados qualitativamente acerca do aspecto estudado em relação ao tema Burn-out.

\section{Resultados e discussão}

Na prática diária da docência de ensino superior, diversos aspectos influenciam o estado emocional e físico da atividade docente e alguns fatores se destacam como geradores de quadros de estresse e possíveis precursores da Síndrome de Burn-out como: ambiente de alta pressão, cultura e política organizacional, baixos salários, perspectiva duvidosa quanto ao crescimento profissional, excesso de carga horária, instabilidade de disciplinas e desinteresse de estudantes. O quadro 1 sintetiza o enfoque abordado por cada um dos artigos incluídos neste estudo, de modo a permitir a análise e agrupamento pelo contexto ao qual ele se refere em relação à Síndrome de Burn-out e o docente de ensino superior.

Quadro 1 - Agrupamento dos artigos por tópico explorado no trabalho de acordo com o enfoque*

\begin{tabular}{|c|c|}
\hline Tópico & Autores \\
\hline $\begin{array}{l}\text { Características } \\
\text { pessoais }\end{array}$ & $\begin{array}{l}\text { YU et al., 2019. CHATANI et al., 2017. HALL; LEE; RAHIMI, 2019. LAL; THARYAN; } \\
\text { THARYAN, 2020. NORI et al., 2019. PADILLA; THOMPSON, } 2016\end{array}$ \\
\hline $\begin{array}{l}\text { Demandas } \\
\text { Laborais }\end{array}$ & $\begin{array}{l}\text { AQUINO et al., 2018. VAN DEN BERG et al., 2015. BRITO LAREDO, } 2018 . \\
\text { HOFFMANN et al., 2017. NASSAR et al., 2020. NASSAR; WAHEED; TUMA, } 2019\end{array}$ \\
\hline Vulnerabilidade & $\begin{array}{l}\text { CASSIDY-VU; BECK; MOORE, 2017. LAL; THARYAN; THARYAN, 2020. EL- } \\
\text { IBIARY; YAM; LEE, 2017. HOFFMANN et al., 2017. NASSAR et al., 2020. REYES- } \\
\text { OYOLA; PALOMINO-DEVIA; APONTE-LÓPEZ, 2019. ALVES; OLIVEIRA; PARO, } \\
2019 .\end{array}$ \\
\hline $\begin{array}{l}\text { Influência do } \\
\text { ambiente }\end{array}$ & $\begin{array}{l}\text { DRAFAHL, 2020. KINSER et al., 2019. ONG et al., 2019. OGLESBY; GALLUCCI; } \\
\text { WYNVEEN, 2020. PADILLA; THOMPSON, 2016. VAN DEN BERG et al., } 2018\end{array}$ \\
\hline $\begin{array}{l}\text { Aspectos } \\
\text { organizacionais }\end{array}$ & DUKE et al., 2020. ROSADO; LORENZO, 2016. \\
\hline Detecção & $\begin{array}{l}\text { BRITO LAREDO, 2018. CHICHRA; ABHIJNHAN; THARYAN, 2019. REYES- } \\
\text { OYOLA; PALOMINO-DEVIA; APONTE-LÓPEZ, 2019. RILEY et al., 2020. EL- } \\
\text { IBIARY; YAM; LEE, 2017. KAVANAGH; SPIRO, 2018. SUMMERS et al., 2019. } \\
\text { BUSTAMANTE et al., 2016. KRUEGER et al., 2017. AGANA et al., 2017. NARVÁEZ } \\
\text { et al., 2017. NAZARI et al., 2016. SHAH et al., 2018. ARVANDI } \text { et al., 2016. OLSON et } \\
\text { al., 2019. ONG } \text { et al., 2019. }\end{array}$ \\
\hline
\end{tabular}


\begin{tabular}{|l|l|}
$\begin{array}{l}\text { Estratégias } \\
\text { prevenção }\end{array}$ & $\begin{array}{l}\text { OWENS, 2017. SAEED et al., 2017. WISENER; EVA, 2018. ABAZA; NELSON, } 2018 . \\
\text { VAN DEN BERG et al., 2017. ALVES; OLIVEIRA; PARO, 2019. BREDELLA; } \\
\text { FESSELL; THRALL, 2019. CRANMER et al., 2018. GONZALEZ; EBERIEL; SHEA, } \\
\text { 2019. LUTHAR, 2017. NARVÁEZ et al., 2017 }\end{array}$
\end{tabular}

*alguns trabalhos se enquadram em mais de um tópico.

Fonte: Autor

A análise dos artigos permitiu categorizar e agrupar a abrangência das publicações acerca dos contextos da Síndrome de Burn-out que afetam os docentes de ensino superior, bem como os aspectos geradores, fatores de risco, situações a serem gerenciadas e estratégias de enfrentamento do problema. A abordagem perpassa vertentes circunstanciais e dinâmicas, contemplando características laborais e individuais e repercutindo em aspectos que, para elucidar o entendimento das dimensões do Burn-out, podem ser analisados à luz dos seguintes contextos: características pessoais, demandas laborais, vulnerabilidade, influência do ambiente, aspectos organizacionais, detecção e estratégias de prevenção.

\section{Características pessoais}

Sabe-se que o trabalho significativo, relacionamentos fortes, estruturas positivas de equipe e a conexão social são essenciais para o bem-estar profissional e pessoal. Os fatores precursores de Burn-out, que incidem sobre a prática do professor podem influenciar estados pessoais e contextos de vida, potencializando-os negativamente. É com frequência que ouvimos relatos de intensificação de quadros de depressão e transtornos de ansiedade devido às situações estressantes vividas no cenário profissional.

Quando pensamos nos aspectos relacionados ao cotidiano pessoal do profissional docente como característica potencializadora ou mitigadora de estresse, além de precursores de saúde mental, devemos considerar os hábitos e manutenção de uma vida saudável. Os estudos de Padilla e Thompson (2016) observam alguns aspectos da exaustão do corpo docente relacionados a rede social de convívio, família, sono e lazer. Pfeffer (2019) relata que, com frequência, as pessoas desconhecem suas reações aos estados de estresse máximo relacionados ao trabalho e quando se deparam com os sentimentos gerados, há falhas em competências emocionais fundamentais para lidar com elas. Essa constatação parte da associação entre o número de casos de afastamentos, suicídio e até mesmo homicídio, com relatos de estresse intolerável ou ambientes tóxicos de trabalho nos Estados Unidos da América, seja na classe de trabalhadores diretos, quanto nas posições de alto escalão de grandes corporações. 
Numa análise da relação entre as prioridades estabelecidas para os planos de vida pessoal e a vida real de docentes universitários, Chatani et al. (2017) evidenciaram um risco aumentado de Burn-out quando há distanciamento entre essas prioridades, destacando que o sistema familiar e o relato de presença de crianças é um tipo de apoio social com efeito mitigador da síndrome na população japonesa. Algumas crenças motivacionais, falha na autorregulação (conceito de autoeficácia e procrastinação) e saúde psicológica no corpo docente, foram testadas nos estudos de Hall, Lee e Rahimi (2019), e Nori et al. (2019), demonstrando o impacto desses três aspectos na evidência causal de Burn-out e aspectos como a empatia e espiritualidade diante do estresse, foram apontados como fontes de apoio para dar mais sentido ao trabalho (LUTHAR, 2017). Yu et al. (2019) acrescentam a essa percepção as características pessoais de autoconceito, mostrando em seu trabalho, que em docentes médicos coreanos, quanto mais positivo o autoconceito profissional, maior a autoestima na profissão e menor o índice de Burn-out.

\section{Demandas laborais}

Ao que se refere ao cotidiano acadêmico, a compreensão do seu papel interfere nos diferentes contextos de atuação no que o professor está inserido e para o qual elabora sua percepção de trabalho. A organização de sua prática diária, em sua totalidade, é expressa pela somatória de trabalhos articulados, o que para o docente de ensino superior, se configura no cumprimento das normativas curriculares das disciplinas designadas a ele. Essas atividades incluem o planejamento, preparação e registros de realização da aula; que devem passar pelo rigor técnico e científico da manutenção do meio acadêmico, com geração e produção de pesquisas científicas; e ainda envolvem os trâmites administrativos do mundo corporativo no desenvolver e organizar produtos de conteúdo científico atualizado por meio de cursos, congressos, seminários, jornadas científicas, entre outros. De fato, sabe-se que o trabalho do docente de ensino superior não se dá de forma isolada, ele mantém elementos interdependentes e acontece de forma dinâmica e complexa.

Petto et al. (2016) destacam, como fatores estressores desse público, os postos de trabalho cada vez mais exigentes, com a necessidade de conciliar o tripé ensino, pesquisa e extensão, acarretando uma carga intensa de atividades. Um corpo docente sobrecarregado refere menos confiança em suas habilidades de ensino e menos hábitos de aprendizagem ao longo da vida acadêmica (NASSAR; WAHEED; TUMA, 2019). O trabalho de Brito Laredo (2018) compreende o entendimento das demandas do trabalho docente como forma de alavancar e 
estruturar propostas de melhoria das práticas educativas e do desenvolvimento e formação dos docentes.

Aquino et al. (2018) mostram o impacto da demanda de trabalho na intenção do profissional em sair da docência, ao que Hoffmann et al. (2017) somam as implicações à saúde do professor universitário e ao desafio em encontrar um equilíbrio entre deveres profissionais, educacionais, de pesquisa e administrativos. Em profissões da saúde, diversos estudos demonstram que a natureza das demandas do trabalho docente na medicina, requerem considerar a junção da prática clínica com o ensino, o que gera alta carga de trabalho, impactando, inclusive, na qualidade da educação oferecida e na segurança do paciente atendido (NASSAR et al., 2020; NASSAR; WAHEED; TUMA, 2019; VAN DEN BERG et al., 2015).

\section{Vulnerabilidade}

Estar vulnerável, implica compreender a condição apresentado por um sujeito que o deixa mais suscetível a um risco diante da exposição à fatores ou ambiente nocivo à sua preservação ou integralidade, seja de ordem física, psicológica, ambiental, social, política. No entendimento do contexto de vulnerabilidade, procuramos os fatores que fragilizam os professores de ensino superior no enfrentamento das situações de estresse aos quais são submetidos na prática docente, para que, diante dessa perspectiva, seja possível repensar os aspectos que podem ser customizados em relação à prevenção e controle dos riscos.

Hoffmann et al. (2017) entrevistaram docentes e identificaram grande vulnerabilidade das mulheres ao esgotamento, identificando sobrecarga cognitiva, danos de ordem física e social, além de comprometimento pessoal, enfatizando que o papel de gênero deve ser considerado enquanto elemento que favorece o adoecimento dos docentes. A mesma vulnerabilidade é citada em outros trabalhos, que enfatizam o impacto negativo da Síndrome de Burn-out na qualidade de vida das mulheres docentes (ALVES; OLIVEIRA; PARO, 2019), sua maior dificuldade na manutenção do equilíbrio entre vida profissional e pessoal (CASSIDY-VU; BECK; MOORE, 2017), sobretudo quando associam a docência e a maternidade com filhos na idade entre 1 e 12 anos, refletindo em sentimento de culpa e desgaste emocional (EL-IBIARY; YAM; LEE, 2017; REYES-OYOLA; PALOMINO-DEVIA; APONTE-LÓPEZ, 2019). Ainda sobre o público com características associadas com maior susceptibilidade ao Burn-out, o trabalho de Lal, Tharyan e Tharyan (2020) aponta alto estresse entre professores mais jovens, ao que Nassar et al. (2020) acrescentam solteiros e docentes em início de carreira como mais propensos para sofrer a Síndrome de Burn-out. 


\section{Influência do ambiente}

Indivíduos que se submetem a um ambiente estressor, muitas vezes têm clareza e compreendem o que lhes afeta, sentem as emoções negativas geradas a partir dos impactos psicológicos ou mesmo físicos que aquele local ou as pessoas que o frequentam, lhe causam. No entanto, existe uma curiosa demora para a procura de auxílio ou mesmo para a valorização do cenário com a importância que ele merece. O estado de colapso com repercussões na saúde é, então, o marcador temporal de que o ambiente se apresenta tóxico o suficiente para comprometer a atividade desenvolvida.

Sentimentos de desgaste, mostram docentes menos motivados ou envolvidos em seu local de trabalho, incluindo nas interações com os alunos e uso da criatividade (DRAFAHL, 2020). O ambiente desafiador de promoção de formas inovadoras de ensinar, a quantidade de carga horária distribuída entre sala de aula e programas de pesquisa, aparecem como motivos de desistência da carreira científica (KINSER et al., 2019) associados às questões de mau relacionamento entre professores, necessidades não atendidas pela gestão direta (ONG et al., 2019), atividades de concessão e demandas não planejadas (PADILLA; THOMPSON, 2016). Van den Berg et al. (2018) enfatizam que a proteção ao desgaste envolve melhorar o ambiente de ensino, ao que Oglesby, Galluci e Wynveen (2020) detalham a consideração dos aspectos que geram conflito entre vida profissional e pessoal, fatores organizacionais, como salários baixos, longas horas de atividade e dificuldades em lidar com a "política e burocracia" da área de atuação.

\section{Aspectos organizacionais}

Ancorando as questões relacionadas à influência do ambiente, a organização e sua forma de gerenciar as atividades docentes e políticas educacionais, repercutem no desgaste laboral e aparecem como fatores condicionantes de estresse crônico, aumentando o status de exaustão. Fatores ambientais e culturais da organização são fontes para a construção ou prejuízo à motivação do docente. O estudo de Duke et al. (2020) vai ao encontro dos achados de Rosado e Lorenzo (2016), referindo-se ao setor educacional como um dos mais expostos e vulneráveis ao sofrimento e sobrecarga de pressões diversas, propondo uma perspectiva transdisciplinar para considerar que os sintomas manifestados pelos docentes se relacionam, muitas vezes, a elementos culturais e organizacionais relativos à carga de trabalho, estrutura proposta e remuneração. A preocupação e empenho em criar locais de trabalho onde pessoas possam prosperar e trabalhar em plenitude, dentro de seus limites físicos e emocionais, gozando de 
saúde clínica e mental, sem enfrentar desgaste ou doenças resultantes de práticas institucionais é o que faz uma empresa ter sustentabilidade humana e preservar seu capital intelectual, bem intangível de seu escopo de funcionamento.

\section{Detecção}

A busca da compreensão sobre o estado emocional dos docentes e suas repercussões na qualidade de vida e na profissão é a base para desmistificar os contextos subjetivos aos quais se incorporam os estados de estresse e, consequentemente, a queda de produtividade e comprometimento da saúde. Para tanto, existem diversas ferramentas que se propõem a detectar variáveis controladas que possam sugerir a ocorrência da Síndrome de Burn-out nos indivíduos estudados. A maior parte dos artigos encontrados nesse estudo, mostram, em algum momento, a intenção de, primeiramente, mensurar o alcance dessa síndrome no público docente para então, destacar os aspectos e influenciadores internos ou externos, assim como analisar alternativas para enfrentamento, prevenção e controle. Compreender a tríade formada pelos aspectos pessoal, profissional e organizacional que incide no trabalho de professores universitários gerando a Síndrome de Burn-out são o ponto de partida de programas de desenvolvimento docente, podendo gerar profissionais mais equipados e preparados para as altas demandas e exigências dos métodos de ensino, melhorando o equilíbrio emocional e abrindo caminho para intervenções efetivas de redução do estresse ocupacional.

\section{Estratégias de prevenção}

A contextualização da Síndrome de Burn-out em docentes universitários abarca campos de ordem pessoal, filosófica, psicológica, cultural e organizacional e ainda são subjetivas as propostas de intervenção e controle, o que pode estar relacionado ao fato de não haver protocolos de conduta para um estado que ainda não é considerado como precursor de uma doença oficialmente declarada e, para o qual, a maioria das pesquisas ainda busca entender as proporções e características.

Wisener e Eva (2018) tratam, com sutileza, que recompensas e incentivos poderiam ser usados de forma efetiva, considerando, para isso, a motivação intrínseca dos envolvidos. Em outra proposta de abordagem preventiva, Bredella, Fessell e Thrall (2019) relatam sobre a estratégia de mentoria como uma proposta para melhora da performance do docente, descrevendo-a como uma alternativa para áreas muito afetadas pelo estresse. Em outro estudo que fundamenta o uso da mentoria como um programa bem-sucedido, Cranmer et al. (2018) 
destacam o uso dela como forma de promover o aumento da produtividade acadêmica, melhor retenção e promoção de docentes e o desenvolvimento de profissionais referência em atendimento, pesquisa, educação e administração. O papel da mentoria colaborativa como potencial para atender às necessidades do corpo docente universitário para aulas práticas, trabalho e prática reflexiva, partilha de conteúdos e conhecimentos pedagógicos, parecem reduzir os fatores geradores do esgotamento (GONZALEZ; EBERIEL; SHEA, 2019).

Algumas sugestões de estratégias de prevenção para os estados estressores e geradores da Síndrome de Burn-out incluem: foco nas intervenções que poderiam fornecer aos professores uma base sólida para o bem-estar e melhora do desempenho em todas as suas funções de trabalho (feedback, avaliação de desempenho, programas de desenvolvimento e reconhecimento) (VAN DEN BERG et al., 2017); promoção de cultura de bem-estar para professores e restauração do equilíbrio (ABAZA; NELSON, 2018); reconhecimento apropriado nas avaliações do corpo docente para aumentar a produtividade, promover a resiliência e mitigar o desgaste no mundo competitivo da academia contemporânea (LUTHAR, 2017); programas com ênfase na resiliência como estratégia para o gerenciamento de estresse e ansiedade, transpondo o modelo de bem-estar para além da atividade profissional, incorporando-o à vida pessoal (SAEED et al., 2017); e avaliação da rede de apoio e suporte familiar, demandas, carga de trabalho e atributos financeiros, implicações de ordem pessoal ou de administração do tempo (OWENS, 2017).

\section{Considerações finais}

A atividade docente é um exercício permeado de desafios corporativos, anseios pessoais, expectativas, exigências técnicas e administrativas tanto na produção de conhecimento quanto no desenvolvimento humano em relação ao aluno e ao futuro profissional em formação para o mercado. Há diversas evidências de que esses fatores, em conjunto, alicerçam estados de abalo emocional, frustração, sobrecarga e preocupações.

A referência de Burn-out como, literalmente, uma "queima até o fim", denota os aspectos que consomem a estrutura produtiva de um trabalhador e, no ambiente acadêmico, nos fomenta a reflexões que extrapolam as paredes das universidades e nos remetem à análise de contextos em diversos cenários, timidamente abordados em políticas organizacionais estruturadas de maneira pouco inclusiva para o docente.

Os dados gerais sobre a Síndrome de Burn-out no Brasil, destacam o país com o segundo lugar no ranking mundial, sendo que a síndrome ainda é considerada como um causador de 
doença e não uma doença propriamente dita, o que tem previsão para acontecer a partir de 2022, quando será incorporada ao Código Internacional de Doenças (CID). As estatísticas, somente então, poderão posicionar cada área efetivamente na ocorrência dos distúrbios associados a ela, o que poderá fundamentar ainda mais as evidências desse mal no professorado de todos os níveis no Brasil.

Ainda poucas são as publicações que abordam a prevenção da síndrome e medidas políticas voltadas para o estabelecimento e auditoria de estratégias educativas que promovam a preocupação com o bem-estar físico e emocional do professor, principalmente devido as características subjetivas que definem a interação entre o profissional e seu trabalho. Ainda, o mais perceptível corresponde a investigação, diagnóstico e classificação no nível de estresse desses profissionais, na busca de entendimento dos eixos precursores e da amplitude da ocorrência da exaustão profissional nessa categoria. Tudo isso demonstra que cabe muito desenvolvimento sobre o tema para que as decisões acerca de como gerenciá-lo repercutam efetivamente na prática docente.

\section{Referências}

ABAZA, Mona M.; NELSON, Kathleen. G. Leading by example: role modeling resilience helps our learners and ourselves. Academic Medicine, Washington, v. 93, n. 2, p. 157-158, 2018. Disponível em: https://pubmed.ncbi.nlm.nih.gov/28991841/. Acesso em: 20 maio 2020.

AGANA, Denny et al. Job satisfaction among academic family physicians. Family Medicine, Leawood Kansas, v. 49, n. 8, p. 622-625, 2017. Disponível em: https://pubmed.ncbi.nlm.nih. gov/28953294/. Acesso em: 18 feb. 2020.

ALVES, Priscila Castro; OLIVEIRA, Aurea de Fatima; PARO, Helena Borges Martins da Silva. Quality of life and Burnout among faculty members: How much does the field of knowledge matter? PLoS ONE, California, v. 14, n. 3, p. 1-12, 2019. Disponível em: https://journals.plos.org/plosone/article/comments?id=10.1371/journal.pone.0214217. Acesso em: 20 maio 2020.

AQUINO, Elizabeth et al. The impact of Burnout on doctorate nursing faculty's intent to leave their academic position: A descriptive survey research design. Nurse Education Today, Inglaterra, v. 69, p. 35-40, 2018. Disponível em: https://www.sciencedirect.com/science/article/abs/pii/S0260691718302818 Acesso: 19 maio 2020 .

ARVANDI, Zeinab et al. Linking medical faculty stress/Burnout to willingness to implement medical school curriculum change: a preliminary investigation. Journal of Evaluation in Clinical Practice, Inglaterra, v. 22, n. 1, p. 86-92, 2016. Disponível em: https://onlinelibrary.wiley.com/doi/pdf/10.1111/jep.12439 Acesso em: 20 maio. 2020. 
BARBOSA, Andrea Loly Kraft Horta. A síndrome de Burnout em professores universitários. 2016. Dissertação (Mestrado em Promoção da Saúde) - Centro Universitário de Maringá, Paraná, 2016. Disponível em: http://rdu.unicesumar.edu.br/handle/123456789/77 Acesso em: 11 abr. 2020.

BATISTA, Jaqueline Brito Vidal et al. Prevalência da Síndrome de Burnout e fatores sociodemográficos e laborais em professores de escolas municipais da cidade de João Pessoa PB. Revista Brasileira Epidemiológica, São Paulo, v. 13, n. 3, p. 502-512, 2010. Disponível em: https://www.scielo.br/scielo.php?script=sci_arttext\&pid=S1415-790X2010000300013 Acesso em: 21 maio 2020.

BAPTISTA, Makilim Nunes et al. Burnout, estresse, depressão e suporte laboral em professores universitários. Revista Psicologia: Organizações e Trabalho, Brasília, v. 19, n. 1, p. 564-570, 2019. Disponível em: http://pepsic.bvsalud.org/scielo.php?script= sci_arttext\&pid=S1984-66572019000100008 Acesso em: 14 jun. 2020.

BERNARDES, Pablo Ferreira. Síndrome de Burn-out: considerações iniciais. In: MENDANHA, Marcos; BERNARDES, Pablo Ferreira; SHIOZAMA, Pedro. Desvendando o Burn-out: uma análise interdisciplinar da síndrome do esgotamento profissional. São Paulo: LTR, 2018. p. 7-11.

BREDELLA, Mirian; FESSELL David; THRALL James H. Mentorship in academic radiology: why it matters. Insights Imaging, Boston, v. 10, n. 1, p. 107, nov. 2019. Disponível em: https://insightsimaging.springeropen.com/articles/10.1186/s13244-019-07992 Acesso em: 19 maio 2020.

BRITO LAREDO, Janette. Calidad educativa en las instituciones de educación superior: evaluación del síndrome de Burnout en los profesores. RIDE Revista Iberoamericana para la Investigación y el Desarrollo Educativo, México, v. 8, n. 16, p. 516-534, 2018. Disponível em: https://www.ride.org.mx/index.php/RIDE/article/view/356 Acesso em: 22 maio 2020.

BUSTAMANTE, Ekaterina et al. El Burnout en la profesión docente: un estudio en la escuela de bioanálisis de la Universidad de Carabobo Sede Aragua, Venezuela. Medicina y Seguridad del Trabajo, Madrid, v. 62, n. 243, p. 111-21, 2016. Disponível: https://scielo.isciii.es/scielo.php?script=sci_arttext\&pid=S0465-546X2016000200003 Acesso em: 5 maio 2020.

CARLOTTO, Mary Sandra. A síndrome de Burnout e o trabalho docente. Psicologia em Estudo, Maringá, v. 7, n. 1, p. 21-29, 2002. Disponível: https://doi.org/10.1590/S141373722002000100005 . Acesso em: 22 maio 2020.

CASSIDY-VU, Lisa; BECK, Keli; MOORE, Justin B. Burnout in female faculty members: A statistic or an opportunity? Journal of Primary Care and Community Health, Estados Unidos, v. 8, n. 2, p. 97-99, 2017. Disponível em: https://www.ncbi.nlm.nih.gov/pmc/articles/PMC5932657/ Acesso em: 11 maio 2020.

CHATANI, Yuki et al. Effects of gaps in priorities between ideal and real lives on psychological Burnout among academic faculty members at a medical university in Japan: A cross-sectional study. Environmental Health and Preventive Medicine, Kyoto, v. 22, n. 1, 
p. 1-7, 2017. Disponível em: https://environhealthprevmed.biomedcentral.com/articles/ 10.1186/s12199-017-0626-7 Acesso em: 19 maio 2020.

CHICHRA, Astha; ABHIJNHAN, Akhil; THARYAN, Prathap. Job stress and satisfaction in faculty of a teaching hospital in south India: A cross-sectional survey. Journal of Postgraduate Medicine, Mumbai, v. 65, n. 4, p. 201-206, 2019. Disponível em: https://www.ncbi.nlm.nih.gov/pmc/articles/PMC6813678/. Acesso em: 20 maio 2020.

CRANMER, Joan M. et al. An adaptable pediatrics faculty mentoring model. Pediatrics, Arkansas, v. 141, n. 5, 2018. Disponível em:

https://pediatrics.aappublications.org/content/pediatrics/141/5/e20173202.full.pdf. Acesso em: 11 maio 2020.

DALAGASPERINA, Patrícia; MONTEIRO, Janine Kieling. Estresse e docência: um estudo no ensino superior privado. Revista Subjetividades, Fortaleza, v. 16, n. 1, p. 37-51, 2016. Disponível em: https://periodicos.unifor.br/rmes/article/view/5154. Acesso em: 20 maio 2020.

DINIZ, Rosa Virgínia; GOERGEN, Pedro L. Educação superior no Brasil: panorama da contemporaneidade. Avaliação, Campinas; Sorocaba, v. 24, n. 3, p. 573-93, dez. 2019. Disponível em: http://www.scielo.br/pdf/aval/v24n3/1982-5765-aval-24-03-573.pdf. Acesso em: 16 mar. 2020.

DRAFAHL, Bridget. The influences Burnout and lack of empowerment have on creativity in nursing faculty. Nursing Education Perspectives, Filadelfia, v. 41, n. 1, p. 33-36, 2020. Disponível em:

https://journals.lww.com/neponline/Abstract/2020/01000/The_Influences_Burnout_and_Lack _of_Empowerment.9.aspx Acesso em: 09 maio 2020.

DUKE, Naomi N. et al. Institutional factors associated with burnout among assistant professors. Teaching and Learning in Medicine, Estados Unidos, v. 32, n. 1, p. 61-70, 2020. Disponível em https://doi.org/10.1080/10401334.2019.1638263 Acesso em: 18 maio 2020.

EL-IBIARY, Shareen Y.; YAM, Lily; LEE, Kelly C. Assessment of Burnout and associated risk factors among pharmacy practice faculty in the United States. American Journal of Pharmaceutical Education, Arlington, v. 81, n. 4, 2017. Disponível em: https://www.ajpe.org/content/81/4/75 Acesso em: 20 mar. 2020.

FERREIRA, Elizabete Cazzolato; PEZUK, Julia Alejandra. Síndrome de Burnout em professores do ensino superior: repercussões na atividade acadêmica. In: ENCONTRO DE ATIVIDADES CIENTÍFICAS, 22, 2019, Londrina. Anais [...]. Londrina: UNOPAR, 2019.

FILIPPI, Marina; BOMFIM, Murilo. Burn-out. Exame. São Paulo: Valongo, 2020.

GONZALEZ, Kimberly A.; EBERIEL, David T.; SHEA, Thomas. B. Collaborative mentoring for retaining secondary biology teachers. Journal of Microbiology \& Biology Education, Washington, v. 20, n. 3, p. 1-6, 2019. Disponível em: https://www.asmscience.org/content/journal/jmbe/10.1128/jmbe.v20i3.1811 Acesso em: 22 abr .2020 .

HALL, Nathan C.; LEE, So Yeon; RAHIMI, Sonia. Self-efficacy, procrastination, and 
Burnout in post-secondary faculty: An international longitudinal analysis. PLoS ONE, California, v. 14, n. 12, p. 1-17, 2019. Disponível em:

https://journals.plos.org/plosone/article?id=10.1371\%2Fjournal.pone.0226716 Acesso em: 29 maio 2020.

HOFFMANN, Celina et al. Psicodinâmica do trabalho e riscos de adoecimento no magistério superior. Estudos Avançados, São Paulo, v. 31, n. 91, p. 257-6, 2017. Disponível em: https://doi.org/10.1590/s0103-40142017.3191019. Acesso em: 30 mar. 2020.

KAVANAGH, Katherine R.; SPIRO, Jeffrey. Faculty wellness: educator burnout among otolaryngology graduate medical educators. Otolaryngology - Head and Neck Surgery, Estados Unidos, v. 158, n. 6, p. 991-994, 2018. Disponível em:

https://doi.org/10.1177/0194599818770647. Acesso em: 19 abr. 2020.

KINSER, Patricia A. et al. Call to action to support the success of midcareer nurse scientists. Nursing Outlook, Estados Unidos, v. 67, n. 3, p. 252-258, 2019. Disponível em: https://www.sciencedirect.com/science/article/abs/pii/S0029655418304883 Acesso em: 19 abr. 2020 .

KRUEGER, Paul et al. Predictors of job satisfaction among academic family medicine faculty: Findings from a faculty work-life and leadership survey. Canadian Family Physician, Canada, v. 63, n. 3, p. 177-185, 2017. Disponível em: https://pubmed.ncbi.nlm.nih.gov/28292815/ Acesso em: 11 fev. 2020.

LAL, Amos; THARYAN, Anna; THARYAN, Prathap. The prevalence, determinants and the role of empathy and religious or spiritual beliefs on job stress, job satisfaction, coping, Burnout, and mental health in medical and surgical faculty of a teaching hospital: a crosssectional survey. Revue de Medecine Interne, Francia, v. 41, n. 4, p. 232-240, 2020. Disponível em: https://e-tarjome.com/storage/panel/fileuploads/2020-0210/1581338192_gh137.pdf Acesso em: 16 jan. 2020.

LUTHAR, Suniya S. Doing for the greater good: what price, in academe? Perspectives on Psychological Science, Washington, v. 12, n. 6, p. 1153-1158, 2017. Disponível em: https://journals.sagepub.com/doi/abs/10.1177/1745691617727863?journalCode=ppsa. Acesso em: 11 abr. 2020.

MASSA, Lilian Dias Bernardo et al. Síndrome de Burnout em professores universitários. Rev. Ter. Ocup., São Paulo, v. 27, n. 2, p. 180-9, 2016. Disponível em: https://www.revistas.usp.br/rto/article/view/104978. Acesso em: 11 maio 2020.

NARVÁEZ, Santiago et al. Human-centered design of an mhealth app for the prevention of Burnout syndrome. Studies in Health Technology and Informatics, Amsterdam, v. 228, n. 4, p. 215-219, 2017. Disponível em: https://ebooks.iospress.nl/publication/44603. Acesso em: 19 maio 2020.

NASSAR, Aussama K. et al. Burnout among Academic Clinicians as It Correlates with Workload and Demographic Variables. Behavioral Sciences, Ontario, v. 10, n. 6, p. 94, 2020. Disponível em: https://macsphere.mcmaster.ca/bitstream/11375/16518/2/MSc_HSED_THESIS_AussamaNas sar_Final.pdf. Acesso em: 19 maio 2020. 
NASSAR, Aussama K.; WAHEED, Abdul; TUMA, Faiz. Academic Clinicians' Workload Challenges and Burnout Analysis. Cureus, California, v. 11, n. 11, p. 1-5, 2019. Disponível em: https://www.cureus.com/articles/24690-academic-clinicians-workload-challenges-andburnout-analysis. Acesso em: 18 maio 2020.

NAZARI, Hedayat et al. The prevalence of job stress and its relationship with Burnout Syndrome among the academic members of Lorestan University of Medical Sciences. Journal of Caring Sciences, Tabriz, v. 5, n. 1, p. 75-84, 2016. Disponível em: https://europepmc.org/article/PMC/PMC4794547. Acesso em: 20 maio 2020.

NORI, Priya. Is Burnout infectious? Understanding drivers of Burnout and job satisfaction among academic infectious diseases physicians. Open Forum Infectious Diseases, Oxford, v. 6, n. 4, p. 1-4, 2019. Disponível em: https://doi.org/10.1093/ofid/ofz092. Acesso em: 13 maio 2020.

OGLESBY, Leslie W.; GALLUCCI, Andrew R.; WYNVEEN, Christopher J. Athletic trainer Burnout: a systematic review of the literature. Journal of Athletic Training, Columbus, v. 55, n. 4, p. 416-430, 2020. Disponível em: https://doi.org/10.4085/1062-6050-43-19. Acesso em: 18 maio 2020.

OLSON, Nathan et al. Faculty assessment of emergency medicine resident grit: a multicenter study. AEM Education and Training, San Antonio, v. 3, n. 1, p. 6-13, 2019. Disponível em: https://doi.org/10.1002/aet2.10309. Acesso em: 22 mar. 2020.

ONG, Andrew Ming-Liang et al. Evaluating the educational environment in a Singaporean residency programme: can we help reduce Burnout rates? Singapore Medical Journal, Singapura, p. 1-20, 2019. Disponível em: https://doi.org/10.11622/smedj.2019094. Acesso em: 11 maio 2020.

OPAS - Organização Pan Americana de Saúde. CID: Burnout é um fenômeno ocupacional. Brasília, 2019. Disponível em:

https://www.paho.org/pt/noticias/28-5-2019-cid-burnout-e-um-fenomeno-ocupacional.

Acesso em: 3 set. 2019.

OWENS, Joan. Life balance in nurse educators: a mixed-methods study. Nursing Education Perspectives, Filadelfia, v. 38, n. 4, p. 182-188, 2017. Disponível em:

https://doi.org/10.1097/01.NEP.0000000000000177 Acesso em: 21 jan. 2020.

PADILLA, Miguel. A.; THOMPSON, Julia. N. Burning out faculty at doctoral research universities. Stress and Health, Sussex Ocidental, v. 32, n. 5, p. 551-558, 2016. Disponível em: https://doi.org/10.1002/smi.2661. Acesso em: 23 fev. 2020.

PETTO, Jefferson et al. Percepção de estresse em docentes do ensino superior. Revista Diálogos Possíveis, Bahia, v. 15, n. 1, p. 70-84, 2016. Disponível em: https://silo.tips/download/percepao-de-estresse-em-docentes-do-ensino-superior Acesso em: 19 mar 2020. 
PFEFFER, J. Morrendo por um salário: como práticas modernas de gerenciamento prejudicam a saúde dos trabalhadores e o desempenho da empresa - e o que podemos fazer a respeito. Rio de Janeiro: Alta Book, 2019.

PRADO, Claudia Elisa Papa. Estresse ocupacional: causas e consequências. Revista Brasileira de Medicina do Trabalho, Americana, v. 14, n. 3, p. 286-289, 2016. Disponível em: https://www.rbmt.org.br/about-the-authors/122/pt-BR. Acesso em: 15 maio 2020.

REYES-OYOLA, Felipe Augusto; PALOMINO-DEVIA, Constanza; APONTE-LÓPEZ, Néstor William. Síndrome de desgaste profesional, índice de masa corporal y otros factores asociados con la labor de profesores de educación física de Ibagué, Colombia. Biomédica, Bogotá, v. 39, p. 537-46, 2019. Disponível em: https://doi.org/10.7705/biomedica.4282. Acesso em: 3 maio 2020.

RILEY, Timothy D. et al. Feasibility of nonanonymous Burnout surveys in a large academic department. Family Medicine, Leawood Kansas, v. 54, n. 4, p. 278-281, 2020. Disponível em: https://doi.org/10.22454/FamMed.2020.471855. Acesso em: 22 mar. 2020.

ROSADO, Lilian; LORENZO, Yudit Orta. La cultura organizacional y el síndrome de desgaste profesional en profesores universitarios. Chakiñan, Revista De Ciencias Sociales Y Humanidades, Ecuador, n. 1, p. 14-30, 2016. Disponível em: https://dialnet.unirioja.es/servlet/articulo?codigo=6294860. Acesso em: 14 fev. 2020.

SAEED, Sophia et al. Building resilience for wellness: a faculty development resource. MedEdPORTAL: the journal of teaching and learning resources, Washington, v. 13, p. 10629, 2017. Disponível em: https://www.mededportal.org/doi/full/10.15766/mep_23748265.10629. Acesso em: 12 mar. 2020.

SANTOS FILHO, José Camilo dos; DIAS, Carmen Lúcia. Profissão acadêmica e scholarship da docência: novo olhar sobre as múltiplas funções do professor universitário. Avaliação, Campinas; Sorocaba, v. 21, n. 3, p. 837-858, 2016. Disponível em: https://doi.org/10.1590/S1414-40772016000300010. Acesso em: 19 maio 2020.

SHAH, Darshana T. et al. Restoring faculty vitality in academic medicine when Burnout threatens. Academic Medicine, Washington, v. 93, n. 7, p. 979-984, 2018. Disponível em: https://journals.lww.com/academicmedicine/Fulltext/2018/07000/Restoring_Faculty_Vitality _in_Academic_Medicine.15.aspx. Acesso em: 21 mar. 2020.

SILVA, Eduardo Pinto. Adoecimento e sofrimento de professores universitários: dimensões afetivas e ético-políticas. Revista Psicologia: Teoria e Prática, São Paulo, v. 17, n. 1, p. 6171, 2015. Disponível em: http://pepsic.bvsalud.org/scielo.php?script=sci_arttext\&pid=S151636872015000100006. Acesso em: 11 nov. 2019.

SUMMERS, Shane M. et al. The prevalence of faculty physician burnout in military graduate medical education training programs: a cross-sectional study of Academic Physicians in the United States Department of Defense. Military Medicine, Gaithersburg, v. 184, n. 9-10, p. 519-527, 2019. Disponível em: https://academic.oup.com/milmed/article/184/910/e522/5426485. Acesso em: 15 fev. 2020. 
VAN DEN BERG, Joost W. et al. A qualitative interview study on the positive well-being of medical school faculty in their teaching role: Job demands, job resources and role interaction. BMC Research Notes, Londres, v. 8, n. 1, p. 1-11, 2015. Disponível em: https://bmcresnotes.biomedcentral.com/articles/10.1186/s13104-015-1393-4. Acesso em: 19 maio 2020.

VAN DEN BERG, Joost W. et al. Work engagement in health professions education.

Medical Teacher, Dundee, v. 39, n. 11, p. 1110-8, 2017. Disponível em:

https://www.tandfonline.com/doi/full/10.1080/0142159X.2017.1359522 Acesso em: 19 maio 2020.

VAN DEN BERG, Joost W. et al. Faculty's work engagement in patient care: Impact on job crafting of the teacher tasks. BMC Medical Education, Londres, v. 18, n. 1, p. 1-12, 2018. Disponível em: https://bmcmededuc.biomedcentral.com/track/pdf/10.1186/s12909-018-1411z.pdf. Acesso em: 21 mar. 2020.

WISENER, Katherine M.; EVA, Kevin W. Incentivizing medical teachers: exploring the role of incentives in influencing motivations. Academic medicine: Journal of the Association of American Medical Colleges, Washington, v. 93, n. 11S, p. 52-59, 2018. Disponível em: https://doi.org/10.1097/ACM.0000000000002383. Acesso em: 23 maio 2020.

YU, Jihye et al. Professional self-concept and Burnout among medical school faculty in South Korea: A cross-sectional study. BMC Medical Education, Londres, v. 19, n. 1, p. 1-6, 2019. Disponível em: https://www.ncbi.nlm.nih.gov/pmc/articles/PMC6612083/. Acesso em: 30 maio 2020. 